\title{
A MAC Protocol with Little Idle Listening for Wireless Sensor Networks
}

\author{
Chaoguang Men ${ }^{1,2}$, Yongqian $\mathrm{Lu}^{1}$, and Dongsheng Wang ${ }^{1,2}$ \\ ${ }^{1}$ Research and Development Center of High Dependability Computing Technology, Harbin \\ Engineering University, Harbin, Heilongjiang, 150001, P.R. China \\ ${ }^{2}$ National Laboratory for Information Science and Technology, Tsinghua University, \\ Beijing, 100084, P.R. China \\ \{menchaoguang, luyongqian\} @hrbeu.edu.cn
}

\begin{abstract}
In wireless sensor networks, energy efficiency is crucial to achieving satisfactory network lifetime. To reduce the energy consumption significantly, a node should turn off its radio as long as possible. We propose a MAC protocol with little idle listening. The sensor node periodically turns on its radio in polling period and checks for a wake-up signal by sampling the energy on the channel. After polling period, the node enters the long sleep period. The data latency is reduced through reserving the channel in polling period. Analyses and simulations reveal that the proposed protocol outperforms S-MAC and LPL in energy conservation and latency.
\end{abstract}

Keywords: wireless sensor networks, protocol, media access control, energy efficient.

\section{Introduction}

Wireless sensor networks (WSN) consists of a large number of distributed nodes with sensing, data processing, and communication capabilities. These nodes are selforganized into a multi-hop wireless network and collaborate to accomplish a common task. As sensor nodes are usually battery-powered, and they are required to operate as long time as possible, so energy efficiency is critical in design of wireless sensor networks [1].

In this paper, we present a MAC protocol with little idle listening, named $L-M A C$, which not only out performs existing energy-efficient MAC protocols on energy conservation, it also mitigates the problem on hidden node interference through adopting a reasonable forwarding delay mechanism. Moreover, it has a better performance at the data latency.

The remainder of this paper is organized as follows: Section 2 discusses related work on energy-efficient of MAC layer in sensor networks. Section 3 describes the proposed protocol, and analyses the latency. Section 4 compares the performance of $L-M A C$ with existing mechanisms via simulations. Section 5 concludes the paper. 


\section{Related Work}

$S-M A C$ is a protocol developed specifically to address energy issues in wireless sensor networks [2]. It uses a simple scheduling scheme to allow neighbors to sleep for long periods and synchronize wakeups. In $S$-MAC, nodes enter sleep mode when a neighbor is transmitting, and segment long packets to avoid costly retransmissions. While the periodic sleep may result in high latency, especially for multi-hop routing algorithms, since all intermediate nodes have their own sleep schedules. The control messages sent by the nodes, which are nearby the border of virtual cluster, are not very useful to hidden terminal problem, because the schedules are not synchronous. Furthermore, the nodes bordering two or more virtual clusters adopt different listen and sleep schedules, so they consume more power than the others and are apt to be disabled. SUA applies a schedule unifying algorithm to unify multiple schedules, which eliminates the influence of bordering nodes [3]. But the schedule unifying algorithm needs a lot of communications to realize it.

Low-power listening is presented in $B-M A C$ [4]. The basic idea of low-power listening is to shift the cost from the receiver to the transmitter by increasing the length of a preamble. If a preamble is detected, the receiver will continue to listen until the start-symbol arriving and the message being properly received. If no preamble, the radio is turned-off again until the next sample.

$T W-M A C$ proposes that energy can be conserved by amortizing the energy cost of communication over multiple packets [5]. In addition, the protocol allows sensors to control the amount of buffered packets since storage space is limited. The disadvantage of this protocol is that a busy tone must be transmitted before every data packet. This leads to energy waste and message latency. The double radios are too complicated for most sensor networks to implement.

$L A S-M A C$ separates channel reservation process and data transmission process [6]. The nodes having data to forward are scheduled during reservation process. The other nodes with no schedule go to sleep to save energy. Only scheduled nodes are awake during scheduled time. The disadvantage of this protocol is that the nodes having data to forward must wait for next duty circle when they overhear interference, but the channel may not always be busy in this duty circle. The unnecessary waiting results in the decrease in throughput.

\section{The MAC Protocol with Little Idle Listening ( $L-M A C)$}

In proposed protocol $L-M A C$, a duty cycle comprises of a polling period and a sleeping period, as shown in Fig.1.

In this MAC protocol, each node only periodically turns on its radio and checks for a wake-up signal by sampling the energy on the channel in polling period. Whether there is a wake-up signal can be determined within $50 \mu \mathrm{s}$ [7]. After this period, the node enters the long sleep period. A not precise synchronization mechanism is adopted, so that all nodes are able to synchronize in sleep period. The closer the distance between nodes is, the more precise the synchronization between them is. Based on these, L-MAC utilizes a spot of control messages for multi-hop channel reservations in polling period, and data messages are transmitted following the control 
messages. The nodes having messages to forward adopt a reasonable forwarding delay mechanism to mitigate the problem on hidden node interference.

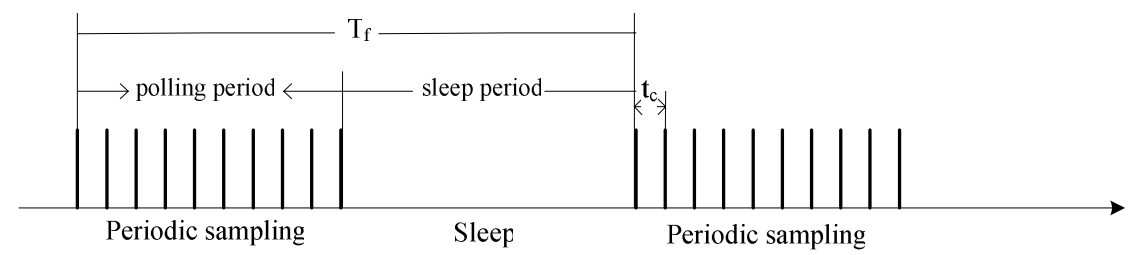

Fig. 1. The L-MAC duty circle

\subsection{Description of L-MAC}

We describe some terms as follow [8]:

- Transmission range. When a node is within transmission range of a sender node, it can receive and correctly decode the packets from the sender node.

- Carrier sensing range. Nodes in the carrier sensing range can sense the sender's transmission. Carrier sensing range is typically much larger than the transmission range [9]. Note that the carrier sensing range and transmission range depend on the transmit power level.

- Carrier sensing zone. Carrier sensing zone is the range which excludes the transmission range from the carrier sensing range. When a node is within the carrier sensing zone, it can sense the signal but can not decode it correctly.

For brevity, we supposed that the carrier sensing range (radius) is one time larger than the transmission range [9].

Fig. 2 denotes the overview of L-MAC. As shown in Fig.2, when a source node (node-a) has data to be sent to the sink node (node-e), it has to wait till the polling period. In polling period, the sender continually sends control messages as preamble to wake up the receiver until the receiver is awoke and decodes the control message correctly. The interval between control messages is denoted by $t_{i}$.

$$
t_{i}=n t_{c}+i t_{m}
$$

In above formula, $n$ is a random natural number, $i$ is the times of retransmission, $t_{c}$ is sampling cycle, $t_{m}$ is the time of control message transmission. When node-a sends the control messages, its second hop neighbor (node-c) which is at the carrier sensing zone of node-a also has chance to sense the wakeup signal [8]. We can say that node-a is likely to help node-b to wake up node-c. This may reduce the control message of node-b. If receiver (node-b) received the control message successfully and it is not the sink node, it will forward the control message to the next hop neighbor (node-c). Node-a takes the control message sent by node-b as the virtual ACK which denotes that node-b has received the control message sent by node-a successfully. Then, if the control message needs to be forwarded further, the process is repeated until the sink node receives the control message successfully and returns an ACK packet. If retransmission times exceed the limit, the sender node will wait for the next polling 
period to retransmit the control message. All the nodes received the control message will keep waking for a period to wait for the data packets. This is a channel reservation process.

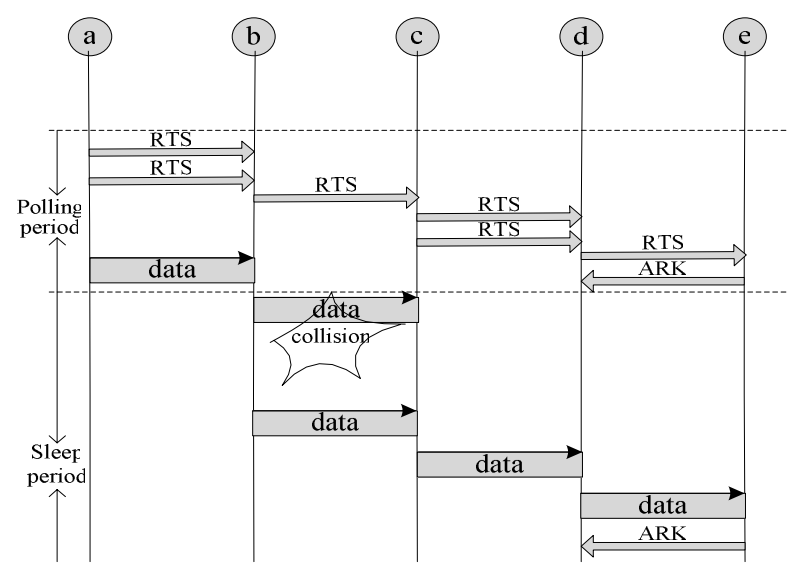

Fig. 2. Overview of L-MAC

The source node (node-a) prepares to send data packets after receiving the control message coming from node-b. The data packets forwarding mechanism can follow the control message forwarding mechanism during channel reservation process.

When a sender senses that the channel is busy, it needs to delay its transmission to mitigate the hidden node interference problem. Otherwise, overlapping transmissions caused by the transmitting node and the hidden node can lead to collision at the first hop node. For example, as shown in Fig.2, when node-a has a data packet to send, it doesn't transmit the packet immediately until node-d has completed the control message forward. Otherwise, overlapping transmissions caused by node-a and node-d can lead to collision at the node-b.

\subsection{Latency Analysis}

We also use the assumptions that adopted in [6]. There are $N$ hops from the source to the sink. For hop $n$, we denote carrier sense delay by $t_{c s, n}$, transmission delay by $t_{t x}$, sleep delay by $t_{s, n}$ and a frame of main duty circle by $T_{f}$.

According to reference [6], the overall delay of a packet over $N$ hops in $S-M A C$ is:

$$
\begin{aligned}
D(N) & =D_{1}+\sum_{n=2}^{N} D_{n} \\
& =t_{s, 1}+t_{c s, 1}+t_{t x}+\sum_{n=2}^{N}\left(T_{f}+t_{c s, n}-t_{c s, n-1}\right) \\
& =t_{s, 1}+(N-1) T_{f}+t_{c s, N}+t_{t x .}
\end{aligned}
$$

So the average latency of S-MAC without adaptive listen over $N$ hops is: 


$$
\begin{aligned}
E[D(N)] & =E\left[t_{s, 1}+(N-1) T_{f}+t_{c s, N}+t_{t x}\right] \\
& =T_{f} / 2+(N-1) T_{f}+t_{c s}+t_{t x} \\
& =N T_{f}-T_{f} / 2+t_{c s}+t_{t x} .
\end{aligned}
$$

Equation (3) shows that the multi-hop latency also linearly increases with the number of hops in $S$-MAC when each node strictly follows its sleep schedules. The slope of the line is the frame length $T_{f}$.

In $L-M A C$, control packets are forwarded ahead to reserve channel. They are always much shorter than data packets. So the forwarding speed must be faster than the data packets. Considering that the polling period is long enough (i.e., 100ms), control message can be transmitted from source node to sink node within the polling period (we will discuss it in section 4). In addition, transmission rate control mechanism makes data packet lag control packet for four hops. In this case, the whole forwarding delay will comprise of three parts: sleep time $t_{s}$, four hops lag $T_{l a g}$ and data packet delay $T_{n}$.

Sleep time $t_{s}$ is the same to $S$-MAC, the mean value is $T_{f} / 2$.

Four hops lag is:

$$
T_{\text {lag }}=4\left(t_{c s}+t_{t x}\right) .
$$

In data packet delay period, all the nodes in the link are awake. When a node receives a packet, it immediately starts carrier sense and tries to forward it to the next hop. The average delay at hop $n$ is $t_{c s, n}+t_{t x}$. The entire data packet delay over $N$ hops is:

$$
D(N)=\sum_{n=1}^{N}\left(t_{c s, n}+t_{t x}\right) .
$$

So the average data packet delay over $N$ hops is:

$$
E[D(N)]=N\left(t_{c s}+t_{t x}\right) .
$$

The total latency of $L-M A C$ is:

$$
\begin{aligned}
E\left[t_{s}+T_{\text {lag }}+D(N)\right] & =E\left[t_{s}\right]+E\left[T_{\text {lag }}\right]+E[D(N)] \\
& =T_{f} / 2+4\left(t_{c s}+t_{t x}\right)+N\left(t_{c s}+t_{t x}\right) \\
& =T f / 2+(N+4)\left(t_{c s}+t_{t x}\right) .
\end{aligned}
$$

We can see that the average latency in $L-M A C$ still linearly increases with the number of hops. Now the slope of the line is $t_{c s}+t_{t x}$. It is much smaller than the slope of $S-M A C$.

\section{Simulations and Performance Evaluation}

We run simulations using OPNET. Except for $L-M A C$, we also implement three MACs for comparison, i.e., a simple version of $S-M A C$ without its synchronization and message passing scheme, $L P L$ and full active 802.11 MAC. The last one will serve as the baselines of latency, energy and delivery ratio. All the sleep scheduled 
protocols have the basic duty cycle of $10 \%$. In $L-M A C$, the polling period is subdivided into 50 sampling periods, and the sampling time is the same as $L P L$ $(50 \mu s)$. The polling period of $L-M A C$ is $100 \mathrm{~ms}$, it is the same long as the listen period of $S$-MAC. We set the bandwidth to $115 \mathrm{kbps}$. Each data packet size is 150 bytes, and control packet size is 15 bytes. 100 nodes are deployed randomly in an area of $300 *$ $300 \mathrm{~m}^{2}$. The radio range (radius) is $30 \mathrm{~m}$. The carrier sensing range (radius) is $65 \mathrm{~m}$. We choose three source nodes and one sink node from different corners. We use the same energy consumption model as in [10] for the radio hardware.

We choose 3 metrics to evaluate the performance of $L-M A C$. Energy cost is the total energy cost to deliver a certain number of packets from sources to sink. This metric shows the energy efficiency of the $M A C$ protocols. Latency is the end to end delay of a packet. Delivery ratio is the ratio of the number of packets arrived at the sink to the number of packets sent by sources.

Fig. 3 shows the averaged packet latency with different hop length. In $L-M A C, L P L$ and full active 802.11 MAC, the latency of them increases with the number of hops. Their increasing exponentials are almost the same. Compared with $L P L$ and 8.2.11 $M A C$, the additional latency of $L-M A C$ comes from the sleep delay at the first hop. $L P L$ only has a short additional preamble at each hop, so its latency gap compared with $802.11 M A C$ is not obvious. As the $S$-MAC protocol has sleep delay at every hop, it has a higher latency.

Fig. 4 shows the energy cost with different hop length in one data stream. In all MAC protocols, the energy cost increases linearly with the number of hops. However, the energy cost of the full active 802.11 MAC increases much faster than other three $M A C$ protocols, because $S-M A C$ has some additional active periods. The additional active periods come from the nodes which are not the receiver but within the overhearing range. $L-M A C$ consumes less energy than $S-M A C$ and $L P L$. The reason is that it has a longer sleep time.

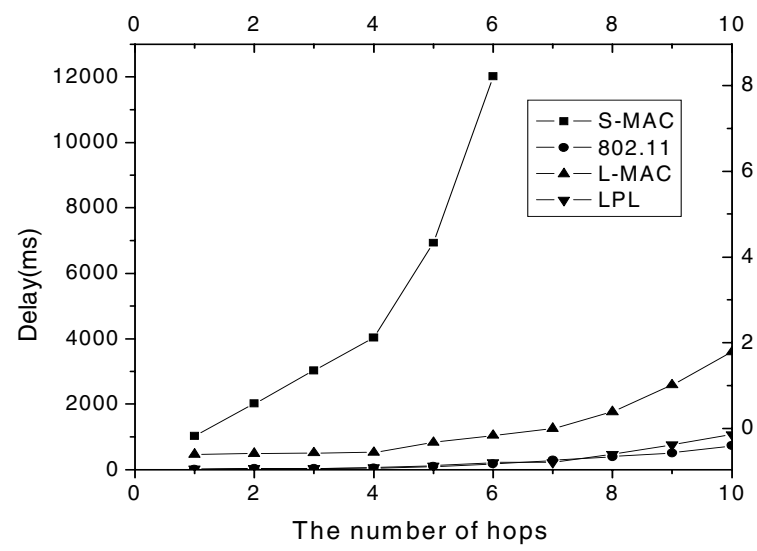

Fig. 3. Mean packet latency on each hop under low traffic load

We then test the traffic adaptation of these MAC protocols, by varying the sensor report interval on the source node from $0.2 \mathrm{~s}$ to $6 \mathrm{~s}$. In all, thirty packets are sent at the source nodes. The simulation time is 1000 s. 
Fig. 5 shows the averaged packet latency for different source report intervals. Clearly, full active 802.11 MAC has the lowest latency. Due to the preamble and the initial sleep delay at the source nodes, $L P L$ and $L-M A C$ have a slightly higher latency. $S-M A C$, however, has much higher latency, especially when traffic load is heavy. The reason is that the packets in $S-M A C$ can be forwarded only one hop during each duty cycle, and packets suffer from both sleep delay and queuing delay. When traffic load is very high, collisions would significantly increase packet latency, because retransmission can only be done after one total duty cycle.

Fig. 6 shows the total energy cost for different source report intervals. On the condition that the total numbers of packets are the same, the energy consumption is almost invariableness. However, the energy cost may slightly increase when the traffic load becoming heavier, it is due to that the retransmission wastes some energy.

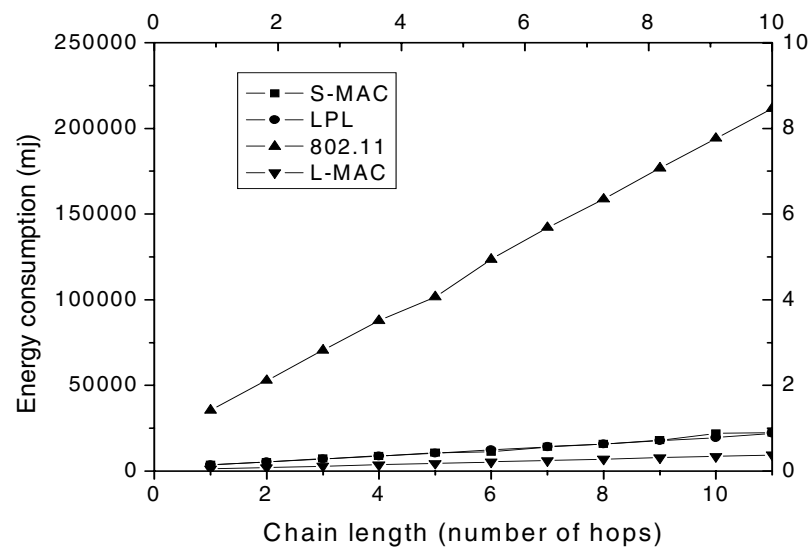

Fig. 4. Total energy consumption on each hop under low traffic load

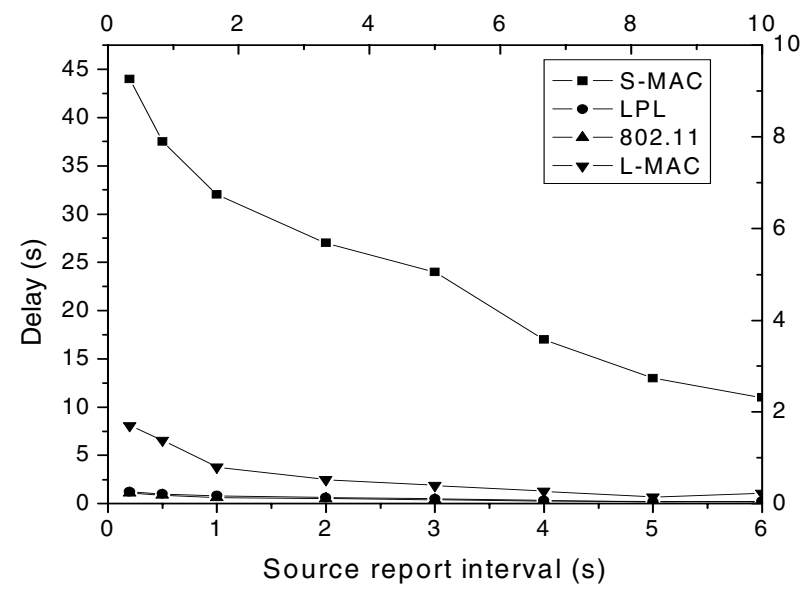

Fig. 5. Mean packet latency under different source report interval 


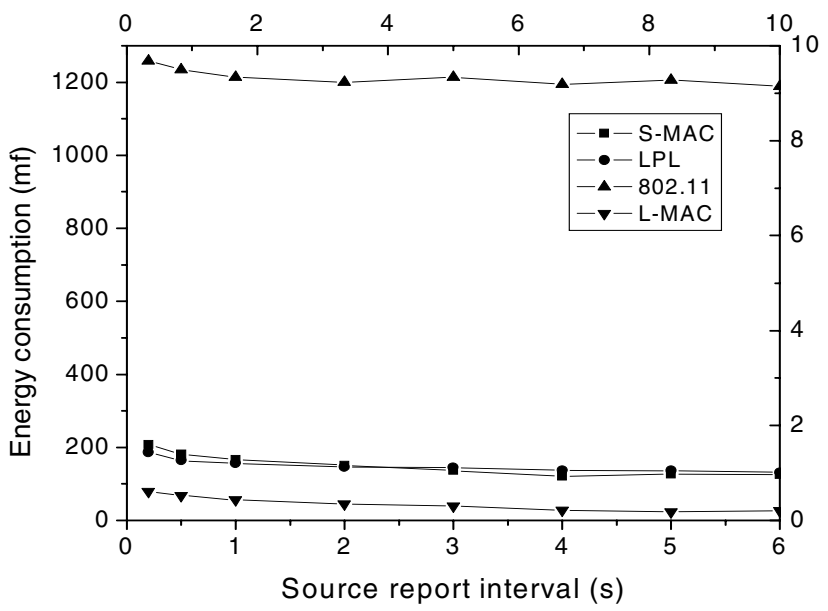

Fig. 6. Energy consumption under different source report interval

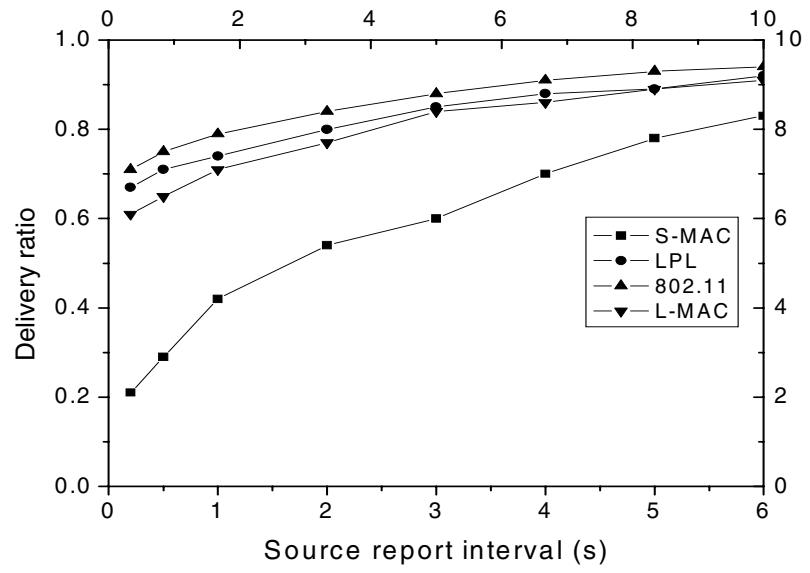

Fig. 7. Throughput under different source report interval

Fig. 7 shows the throughput for different MAC protocols. 802.11 MAC and LPL have the best data throughput. $L-M A C$ also has a better performance. Compared with full active 802.11 MAC, it is good enough for wireless sensor networks.

\section{Conclusions}

This paper presents a novel MAC protocol for wireless sensor networks. Compared to $S-M A C$ and $L P L$, it makes following improvements: minimizing the idle listening, accelerating the data passing, adopting a powerful forwarding delay mechanism to 
mitigate the hidden node interference problem. The simulation results reveal that our protocol has better performance in packet delivery ratio, latency and energy efficiency.

\section{References}

1. Demirkol, I., Ersoy, C., Alagoz, F.: MAC Protocols for Wireless Sensor Networks: A Survey. IEEE Communications 44(4), 115-121 (2006)

2. Ye, W., Heidemann, J., Estrin, D.: Medium Access Control with Coordinated Adaptive Sleeping for Wireless Sensor Networks. IEEE/ACM Transactions on Networking 12(3), 493-506 (2004)

3. Lee, W., Lee, D., Lee, H.S.: Life Extension of Border Nodes in SMAC-based Wireless Sensor Networks by Unifying Multiple Sleep Schedules among Adjacent Virtual Clusters. PE-WASUN, Canada. vol. 10, pp. 267-268 (2005)

4. Polastre, J., Hill, J., Culler, D.: Versatile Low Power Media Access for Wireless Sensor Networks. In: SenSys 2004, Maryland, USA, vol. 11, pp. 95-107 (2004)

5. Miller, M.J., Vaidya, N.H.: A MAC Protocol to Reduce Sensor Network Energy Consumption Using a Wakeup Radio. IEEE Transactions on Mobile Computing 4(3), 228-241 (2005)

6. Kim, J., Park, K., Shin, J.-H., Park, D.: Look-Ahead Scheduling For Energy-Efficiency and Low-Latency in Wireless Sensor Networks. In: PE-WASUN 2006, Spain, vol. 10, pp. 141-144 (2006)

7. Hill, J.L., Culler, D.E.: Mica: A Wireless Platform for Deeply Embedded Networks. IEEE Micro 22(6), 12-24 (2002)

8. Jung, E.-S., Vaidya, N.H.: A Power Control MAC Protocol for Ad Hoc Networks. In: Proceedings of the IEEE/ACM MobiCom Conference, vol. 9, pp. 36-47 (2002)

9. Kamerman, A., Monteban, L.: WaveLAN-II: A High-Performance Wireless LAN for The Unlicensed Band. Bell Labs Technical Journal 2(3), 118-133 (1997)

10. Lin, P., Qiao, C., Wang, X.: Medium Access Control with a Dynamic Duty Cycle for Sensor Networks. In: WCNC 2004/IEEE Wireless Communications and Networking Conference, vol. 3, pp. 1534-1539 (2004) 